\title{
Enhanced tagging of light utilizing acoustic radiation force with speckle pattern analysis
}

\author{
Ali Vakili \\ Joseph L. Hollmann \\ R. Glynn Holt \\ Charles A. DiMarzio
}




\title{
Enhanced tagging of light utilizing acoustic radiation force with speckle pattern analysis
}

\author{
Ali Vakili, ${ }^{a, *}$ Joseph L. Hollmann, ${ }^{a}$ R. Glynn Holt, ${ }^{c}$ and Charles A. DiMarzio ${ }^{a, b}$ \\ ${ }^{a}$ Northeastern University, Computer and Electrical Engineering, Boston, Massachusetts, United States \\ ${ }^{b}$ Northeastern University, Mechanical and Industrial Engineering, Boston, Massachusetts, United States \\ 'Boston University, Mechanical Engineering, Boston, Massachusetts, United States
}

\begin{abstract}
In optical imaging, the depth and resolution are limited due to scattering. Unlike light, scattering of ultrasound (US) waves in tissue is negligible. Hybrid imaging methods such as US-modulated optical tomography (UOT) use the advantages of both modalities. UOT tags light by inducing phase change caused by modulating the local index of refraction of the medium. The challenge in UOT is detecting the small signal. The displacement induced by the acoustic radiation force (ARF) is another US effect that can be utilized to tag the light. It induces greater phase change, resulting in a stronger signal. Moreover, the absorbed acoustic energy generates heat, resulting in change in the index of refraction and a strong phase change. The speckle pattern is governed by the phase of the interfering scattered waves; hence, speckle pattern analysis can obtain information about displacement and temperature changes. We have presented a model to simulate the insonation processes. Simulation results based on fixed-particle Monte Carlo and experimental results show that the signal acquired by utilizing ARF is stronger compared to UOT. The introduced mean irradiance change (MIC) signal reveals both thermal and mechanical effects of the focused US beam in different timescales. Simulation results suggest that variation in the MIC signal can be used to generate a displacement image of the medium. $\odot 2017$ Society of Photo-Optical Instrumentation Engineers (SPIE) [DOI: 10.1117/1.JBO.22.10.106004]
\end{abstract}

Keywords: laser speckle; fixed-particle Monte Carlo; ultrasound guidestar; acoustic radiation force; ultrasound tagging of light. Paper 170323RR received May 20, 2017; accepted for publication Aug. 21, 2017; published online Oct. 6, 2017.

\section{Introduction}

Optical imaging modalities are widely being utilized to generate images with good resolution and contrast in biomedical imaging. However, in optical imaging the depth and resolution are limited due to scattering, even where the absorption of light is low. Unlike light, ultrasound (US) waves can travel through tissue with negligible scattering; hence, they can provide information from deeper parts of the tissue compared to light. However, US images suffer from low contrast compared to optical images.

To use the advantages of both imaging modalities, hybrid imaging methods such as photoacoustic tomography (PAT) have been introduced. PAT utilizes the US to monitor the optical absorption to generate an image. ${ }^{1-5}$ In comparison to PAT, US modulated optical tomography (UOT) can be used to determine both optical absorption and scattering. ${ }^{6-9}$ In UOT, the US wave modulates the local index of refraction and the location of scatterers; therefore, the phases of the scattering paths that pass through the focused US beam are modulated at the US frequency. ${ }^{10-15}$ Therefore, the focused US beam can be utilized as a "guidestar" to modulate scattered light and spatially encode information by tagging the light that passes through the US focal spot. ${ }^{16-19}$

The challenge in UOT is detecting the small signal coming from the modulated light compared to the large amount of unmodulated light, typically requiring expensive equipment for detection.

In order to obtain a stronger signal by acoustically tagging light, other effects of focused US wave can be utilized. ${ }^{20-22}$
Distortion of the scattering medium induced by the acoustic radiation force (ARF), causes greater displacement, and correspondingly greater change in the phase of the scattering paths tagged by the US wave, resulting in a stronger signal, which leads to easier detection. The signal can be used to monitor displacement induced by the ARF and displacement image generation.

Acoustic radiation force impulse (ARFI) imaging utilizes $\mathrm{ARF}$ to induce a mechanical perturbation to the tissue and monitor the tissue response to form the image. ${ }^{23}$ The amount of displacement corresponding to the ARF reveals mechanical properties of the target and forms the basis of different modalities of elastography. ${ }^{22,24}$

ARFI imaging utilizes US detection to monitor the deformation. Speckle pattern analysis is another useful method to monitor tissue deformation. Since the speckle pattern is governed by the location of scattering particles and the index of refraction of the medium, speckle pattern analysis can provide information about scatterers motion in the medium. Particle motion changes the optical path length (OPL) of the light, resulting in a change in the speckle pattern. In addition to the ARF deformation, absorbed acoustic energy generates heat and increases the temperature of the medium, resulting in change in the local index of refraction, which similarly causes strong phase change ${ }^{25-27}$ that can be monitored by speckle pattern analysis. Experimental measurements of ARF also include this thermal effect.

The speckle pattern generated by the reflected light from a surface reveals information about the roughness of the surface. ${ }^{28}$ Analysis of speckle displacement caused by surface deformation is used in surface strain measurement. ${ }^{29}$ In an optically turbid 
medium, speckle pattern analysis can be a useful method to monitor internal changes such as displacement induced by the ARF.

In this paper, we present a two-dimensional (2-D) fixedparticle Monte Carlo to model speckle variation caused by ARF displacement and temperature rise induced by a focused acoustic wave inside an optically scattering medium. Results from the model explain our experimental results of speckle pattern analysis with four different US exposure times. The displacement caused by thermal expansion and change in the local index of refraction due to pressure variation is smaller and thus neglected in our model. We present an analysis based on OPL variation due to the ARF displacement and temperature rise individually. Simulation results presented are based on a fixed-particle Monte Carlo ${ }^{30}$ method. Fixed-particle Monte Carlo is used since the conventional Monte Carlo method does not provide the coherence required in speckle pattern formation. Experimental results for different US pulse widths prove that the signal acquired by utilizing ARF is stronger compared to UOT. Also, displacement induced by the ARF and thermal effect of the focused US wave occur in different time scales. Simulation and experimental results suggest that the introduced mean irradiance change (MIC) signal represents different insonation processes and it can be utilized to monitor the displacement and thermal effects induced only by the ARF. It can also be used to form an image based on the displacement of scatterers in the medium.

\section{Theory}

Particle displacement is the result of the acoustic interaction between an absorbing target with the US wave. As the wave hits the target, the target absorbs part of the power of the wave. The result of this process is wave momentum transfer to the target, which generates the $\mathrm{ARF}^{31-34}$

$F=\frac{2 \alpha \tilde{I}_{0}}{c_{u s}}(\mathrm{~N})$

where $\tilde{I}_{0}$ is the averaged US temporal intensity, $\alpha$ is the acoustic absorption coefficient, and $c_{u s}$ is the speed of US wave propagation.

In addition, in an acoustically absorbing medium, the absorbed energy dissipates and converts into heat. As temperature rises, the local index of refraction of the medium decreases. Therefore, the phase of the scattering path passing through the US beam focal spot is tagged by the US beam. Due to thermal conductivity, the heat spreads in the medium, resulting in cooling the original heated region and heating the surrounding region. Therefore, compared to the ARF displacement, the increase in the temperature affects a larger region of the medium (i.e., a larger number of scattered waves are tagged).

The displacement is a function of the US intensity, time, and medium properties as ${ }^{25,35}$

$\Delta l(\vec{r}, t)=\frac{2 \alpha}{c_{u s}} \tilde{I}(\vec{r}) h(t)(\mathrm{m})$,

and the change in the local index of refraction is a function of change in the local temperature ${ }^{36}$

$\Delta n(\vec{r}, t)=f(\Delta T(\vec{r}, t))$ where the temperature rise is a function of distance to the US focal spot, time, US intensity, and medium properties. The temperature change is ${ }^{25,35}$

$\Delta T(\vec{r}, t)=\frac{2 \alpha}{\rho C_{v}} \tilde{I}(\vec{r}) g(t)(\mathrm{K})$,

where $\vec{r}$ is the location vector from the US focal spot, $\alpha$ is the amplitude absorption coefficient, $\tilde{I}$ is the spatial-peak acoustic intensity, $\rho$ is the medium density, $c_{u s}$ is the speed of sound, $C_{v}$ is the specific heat of the medium, and $h(t)$ and $g(t)$ are the time dependent parts of the displacement and the change in temperature, respectively.

The analysis of the changes in the speckle pattern is utilized to study the ARF effects. As light from a monochromatic light source travels through a highly scattering medium, it undergoes many scattering events, generating different scattering paths. Differences in the OPLs imply different phases for each wave. At the detector, these temporally coherent waves with different phases can destructively or constructively interfere to generate a pattern of bright and dark regions, known as a speckle pattern. The speckle pattern is governed by the phase of the incident waves on the detector, i.e., the OPL of the scattered waves. ${ }^{37,38}$

OPL is defined as the product of the geometric distance that light travels $(l)$ and the index of refraction of the medium $(n)$,

$\mathrm{OPL}=\ln (\mathrm{m})$

We are interested in the changes in the speckle pattern, which is governed by the changes in the OPL of the interfering waves at the detector. Change in the OPL is the result of physical change in the distance between scattering particles and/or change in the local index of refraction of the medium,

$\Delta \mathrm{OPL}=n \Delta l(\tilde{I}, t)+l \Delta n(T(\tilde{I}, t))(\mathrm{m})$,

where $\Delta n$ is the change in the local index of refraction induced by the temperature change and $\Delta l$ is the change in the physical distance between particles due to displacement induced by the ARF. Therefore, the change in the phase of the $i$ 'th wave is

$\Delta \theta_{\mathrm{i}}=\frac{2 \pi}{\lambda} \Delta \mathrm{OPL}_{\mathrm{i}}(\mathrm{rad})$

The resulting electric field for each scattering path after leaving the medium is

$E_{i}=\alpha_{i} e^{j\left(\omega t+\theta_{i}\right)}(\mathrm{V} / \mathrm{m})$

where $\omega$ is the radial frequency of the optical wave. $\alpha_{i}$ and $\theta_{i}$ are the amplitude and the phase of the $i$ 'th path, respectively.

The detector measures the irradiance of the interference of all the waves on a specific pixel as

$I=\frac{c \epsilon_{0}}{2}\left|\sum_{i=1}^{N} \alpha_{i} e^{j\left(\omega t+\theta_{i}\right)}\right|^{2}\left(\mathrm{~W} / \mathrm{m}^{2}\right)$,

where $c$ is the speed of light, $\epsilon_{0}$ is the free space electric permittivity, $N$ is the number of paths contributing in the interference, and $\theta_{i}$ is the phase of the $i$ 'th path. 
Table 1 Original state and four consecutive insonation processes.

\begin{tabular}{lccccc}
\hline & $\begin{array}{c}\text { Original } \\
\text { state }\end{array}$ & $\begin{array}{c}\text { ARF } \\
\text { excitation }\end{array}$ & Heating & $\begin{array}{c}\text { ARF } \\
\text { relaxation }\end{array}$ & Cooling \\
\hline Displacement & 0 & $0 \rightarrow D_{\max }$ & $D_{\max }$ & $D_{\max } \rightarrow 0$ & 0 \\
Temperature & $T_{0}$ & $T_{0}$ & $T \rightarrow T_{\max }$ & $T_{\max }$ & $T \rightarrow T_{0}$ \\
\hline
\end{tabular}

Hence, changes in the irradiance can be calculated as

$$
\begin{aligned}
\Delta I= & \frac{c \epsilon_{0}}{2}\left\{\left.|| \sum_{i=1}^{N} \alpha_{i} e^{j\left[\omega t+\theta_{(1, i)}\right]}\right|^{2}\right. \\
& \left.-\left|\sum_{i=1}^{N} \alpha_{i} e^{j\left[\omega t+\theta_{(1, i)}+\Delta \theta_{i}\right]}\right|^{2} \mid\right\}\left(\mathrm{W} / \mathrm{m}^{2}\right),
\end{aligned}
$$

where $\theta_{(1, i)}$ is the phase of the $i$ 'th path at the original state (state $\# 1)$ and $\Delta \theta_{i}$ represents the changes in the phase of the $i$ 'th path, induced by the US wave. The distribution of $\Delta \theta_{i}$ is important in our analysis, we are going to use the term phase distribution to address this later in the paper.

The total changes in the phase of the scattering paths include changes caused by both displacement $(\Delta l)$ and the changes in the index of refraction $(\Delta n)$.

As the US pulse is turned on and off, the phase distribution goes through four states. We define the state before the insonation as the original state, shown in Fig. 4 as state \#1. Hence, the insonation process can be described in four separate and consecutive processes (Table 1):

- ARF excitation: At the beginning of the insonation, the ARF induced by the US wave causes a perturbation in particles' locations. This process happens faster than our image acquisition speed (62.5 fps); therefore, we observe this process as an instantaneous change in the speckle pattern. It is assumed that no temperature change occurs during ARF excitation process. In the simulation, we do not have such a limitation, but in order to simulate our experiments, we model this process as instant displacement in the medium.

- Heating: After the maximum displacement is reached, the absorbed acoustic energy converts to heat and increases the temperature. It is assumed that no displacement occurs during heating process.

- ARF relaxation: After insonation, in the absence of the ARF, particles move back to their original fixed location. This process also happens faster than our image acquisition speed. Therefore, we can observe this process as an instantaneous change in the speckle pattern, and similar to ARF excitation process, it is assumed that no temperature change occurs during ARF relaxation process.

- Cooling: After all particles move back to their original location, temperature decreases to the temperature at the original state, due to the thermal diffusivity. Since US is turned off, no displacement occurs during the cooling process.

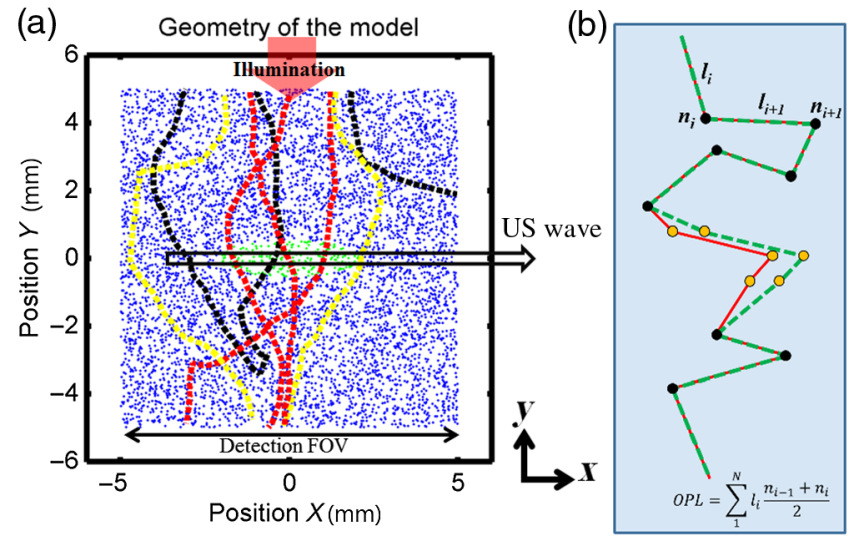

Fig. 1 (a) The 2-D model of the scattering medium. Blue dots represent optical scatterers that are randomly located. The light from the laser enters the medium from the top side and the detection point is set to be at the bottom side of the medium. Green dots are the scatterers affected by the acoustic wave. Red paths: Scattering paths that travel through the acoustic focal area and contribute in the speckle pattern. Yellow paths: Scattering paths that are not affected by the acoustic wave but contribute in the speckle pattern. Black paths: Scattering paths that do not contribute in the speckle pattern. In this model, the acoustic wave propagates in the direction is shown by black arrow (in the direction of positive $x$ ). (b) 2 states of a scattering path, before ARF (solid red line) and after ARF displacement (dotted green line). Yellow particles are the particles affected by the US. Other particles assumed to stay at their original location. Based on the local temperature, each scatterer is assigned an index of refraction, which is used to calculate the OPL of the scattering path.

\section{Model}

The model contains randomly located particles in two dimensions. The size $($ diameter $=10 \mu \mathrm{m})$, index of refraction $(n=1.34)$, and the concentration of the scattering particles (625 scatterer per squared millimeter) determine the optical scattering coefficient of the model. In our 2-D model, the locations of particles are assumed to be fixed in each state of the simulations. This is a valid assumption considering the speed of light compared to the speed of the acoustic wave. Figure 1 represents the geometry of a $1 \mathrm{~cm} \times 1 \mathrm{~cm}$ model.

Functions $h(t)$ and $g(t)$ in Eqs. (2) and (4) are the time-dependent parts of the equations. Since the function $h(t)$ is not well formulated in the literature, to model the perturbation in the location of the particles induced by the ARF, particles are moved in the direction of the acoustic wave propagation (along the $x$ direction in the model). The amount of displacement for each particle depends on the particle's location with respect to the US focal spot. For instance, a particle closer to the focal spot of the US wave moves more than another particle farther away from the US beam. Displacement is modeled as a 2-D Gaussian distribution as a function of position for the entire model; however, the effective displacement occurs in the region of focus.

$\Delta d(x, y)=D_{\max } e^{-\left[\frac{\left(x-x_{0}\right)^{2}}{2 \sigma_{x}^{2}}+\frac{\left(y-y_{0}\right)^{2}}{2 \sigma_{y}^{2}}\right]}(\mathrm{m})$

where $D_{\max }$ is the maximum displacement that occurs at the focal spot. $x$ and $y$ represent position of the particle. $x_{0}$ and $y_{0}$ represent the location of the US focal spot. $\sigma_{x}$ and $\sigma_{y}$ determine the effective size of the US focal spot and $\Delta d$ shows the 
amount of the unidirectional displacement that occurs for the particle located at $x$ and $y$ caused by the ARF. The parameters for the 2-D Gaussian distribution are set so that the effective displacement region models the focal area of the US beam (5 mm axially, $0.5 \mathrm{~mm}$ laterally). The displacement will result in compression of particles at one side of the US focal spot and dilation on the other side.

The change in the temperature due to acoustic wave absorption is a function of the distance to the focal spot of the focused acoustic wave and time (US wave pulse width and time for cooling process). During insonation, we have the temperature rise modeled as ${ }^{35}$

$\Delta T(r, t)=\frac{2 \alpha \tilde{I}_{0}}{\rho C_{v}} e^{-\frac{r^{2}}{a^{2}}} \frac{a^{2}}{4 \tilde{k}} \ln \left(1+\frac{4 \tilde{k} t}{a^{2}}\right)(\mathrm{K})$.

After insonation, we have ${ }^{35}$

$\Delta T(t)=\frac{T_{\text {peak }}}{\left(1+\frac{4 \tilde{k} t}{a^{2}}\right)}(\mathrm{K})$,

where $r$ is the radial distance to the US focal spot, $\alpha$ is the amplitude absorption coefficient, $\tilde{I}_{0}$ is the spatial-peak temporal-average acoustic intensity, $a$ is the effective Gaussian radius of the radial spatial intensity profile, $\tilde{k}$ is the medium thermal diffusivity, $\rho$ is the medium density, $C_{v}$ is the medium specific heat, and $T_{\text {peak }}$ is the temporal-peak temperature reached before cooling, given by Eq. (12). In both cases, $\Delta T$ is defined as the change in the temperature compared to the temperature before insonation $\left(T_{0}\right)$.

In order to model the variation in the speckle pattern, it is required to generate optical scattering paths that interfere at the detector. In conventional Monte Carlo, every time a new photon is launched in the medium, it sees a completely different medium since the travel distance between two scattering event is chosen randomly. Therefore, the conventional Monte Carlo method cannot provide the coherence that is required for speckle pattern formation. To overcome this problem, we have utilized the Monte Carlo method with fixed particles to obtain the coherence in our simulations. ${ }^{30,39}$ In fixed-particle Monte Carlo, after each collision, the scattering angle is chosen randomly based on a weighted probability distribution given by the Mie scattering theory. ${ }^{40}$ Based on the particles' location and the scattering angle, the next scattering event is determined. This process is followed until light either gets absorbed or reaches the boundary of the medium and leaves the medium without further scattering. Figure 1 illustrates a few example scattering paths that have been obtained by the fixed-particle Monte Carlo method. The red paths go through the acoustic wave focal area (shown in green); therefore, their OPL is affected by the acoustic wave (tagged paths). Yellow paths contribute to the speckle pattern formation but are not affected by the US wave (background paths), and black paths do not contribute to the speckle pattern formation. In our simulations, 500,000 scattering paths are obtained and used. For each state of the simulation, the same set of scattering paths is used. Therefore, information of each path is recorded to be used throughout the simulations. To model the optical interference at the detector, the phase determined by the OPL of each optical path is required.

\section{Methods}

Figure 2 represents our experimental setup to capture the laser speckle pattern. The unfocused $\mathrm{CW}$ laser (HeNe laser, $\lambda=$ $632.8 \mathrm{~nm}$ with about $0.3 \mathrm{~mm}$ waist) beam goes through the phantom submerged in degassed water and is scattered to generate the speckle pattern at the detector (Basler-acA192025um—pixel size: $2.2 \mu \mathrm{m} \times 2.2 \mu \mathrm{m}$-no optical filtering). It is important to have the speckle size larger than the detecting pixel to be able to see the changes in the speckle pattern. The distance from the medium to the detector is set so that the size of the speckle $(d)$ is $\sim 5$ times the pixel size on the detector, using ${ }^{38,41,42}$
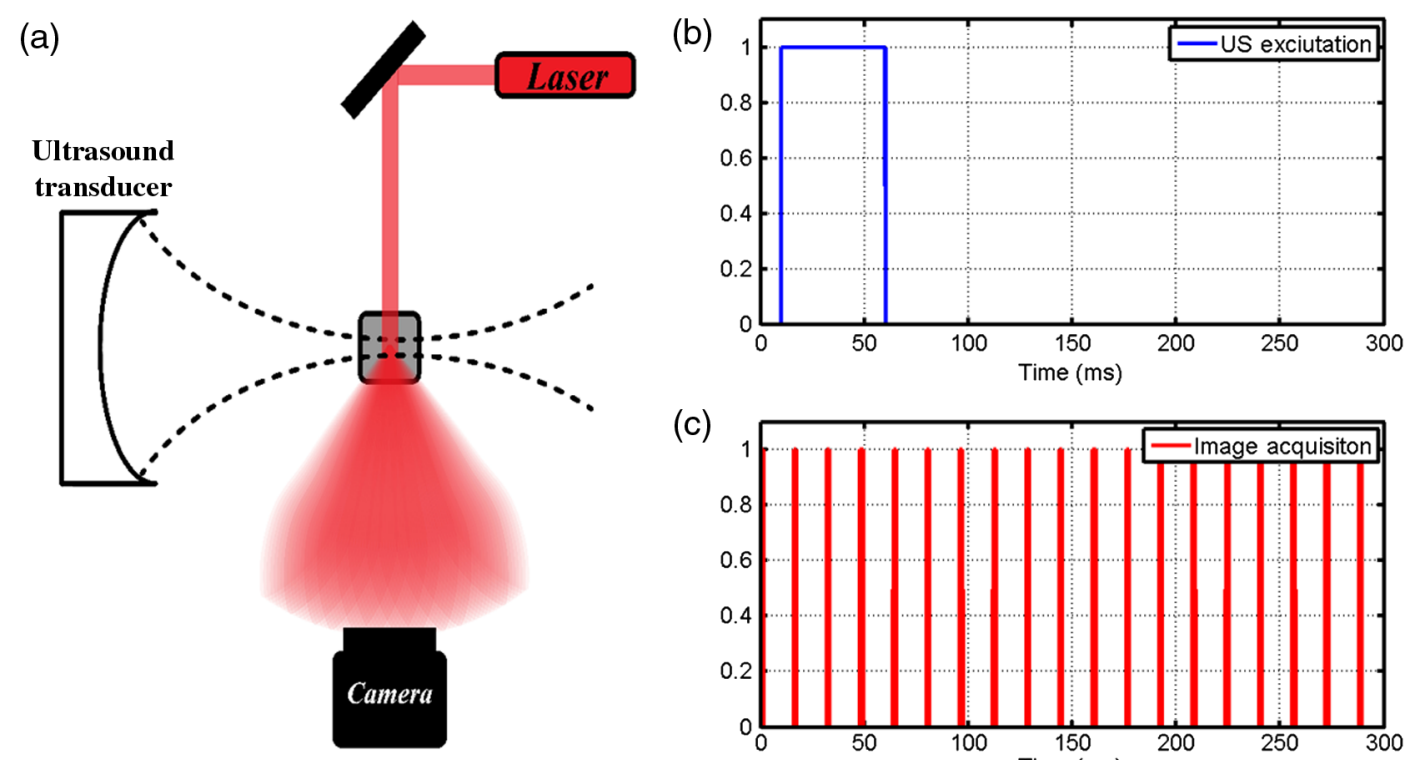

(c)

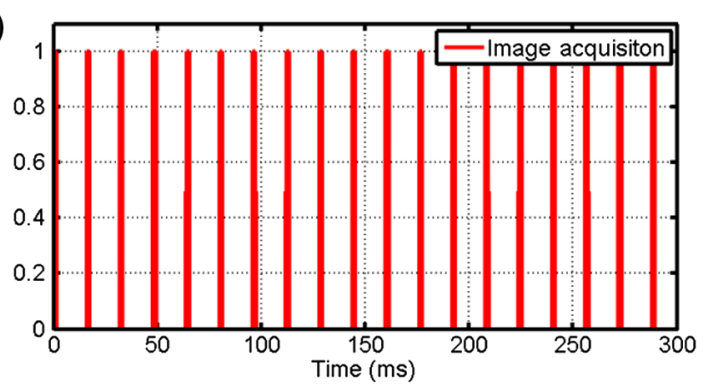

Fig. 2 (a) Experimental setup. The laser is on one side of the phantom. Scattered light leaves the phantom from the other side and generates a speckle pattern on the detector. The US wave is delivered to the phantom perpendicular to the laser and induces displacement perpendicular to the laser direction. (b) US excitation. (c) Image acquisition timing. An image is taken every $16 \mathrm{~ms}$ with $1 \mathrm{~ms}$ exposure time. 
$d=\frac{\lambda}{\mathrm{FOV}} z(\mathrm{~m})$

where $d$ is the speckle size, $z$ is the distance to the detector $(20 \mathrm{~cm})$, FOV represents the field of view where the light is collected from (the $1 \mathrm{~cm}$ side of the phantom), and $\lambda$ is the wavelength of the light source.

The tissue mimicking phantom is made of gelatin to imitate acoustic properties of soft tissue. In order to generate optical scattering, milk is added to the phantom. ${ }^{43}$ The concentration of the milk governs the optical scattering coefficient of the phantom (100 ml water, $5 \mathrm{ml}$ milk, and 20 gr gelatin). The gelatin mixture is degassed before curing in order to minimize the cavitation effect. A focused US transducer (Sonic Concepts, Inc.Model H102) with $f=1.1 \mathrm{MHz}$ (bandwidth $= \pm 250 \mathrm{KHz}$ ) and peak pressure of $2.78 \mathrm{MPa}$ is used to generate the ARF within the phantom. The source focuses to an area with lateral radius about $0.5 \mathrm{~mm}$ and axial radius about $5 \mathrm{~mm}$.

The displacement process occurs in a timescale of milliseconds, while the thermal effect occurs in a timescale of seconds. In order to distinguish the ARF and thermal effects, we have applied US waves with four different pulse widths $(50 \mathrm{~ms}$, $100 \mathrm{~ms}, 1 \mathrm{~s}$, and $2 \mathrm{~s}$ ). An image is captured every $16 \mathrm{~ms}$ (62.5 fps). Figures 2(b) and 2(c) represent image acquisition and US wave excitation timing diagram. Images taken before insonation are set as the original state of the speckle pattern. The difference image is defined as the absolute value of the change in the speckle pattern at any time, compared to the speckle pattern at the original state. We are interested in the changes in the irradiance with respect to time and with the presence of the acoustic wave. Hence, the MIC signal at any given time is defined as the change in the speckle pattern from the original state $(t=0)$ as

$\operatorname{MIC}(t)=\langle|I(x, y, t)-I(x, y, 0)|\rangle$,

where $I(x, y, 0)$ is defined as the original state of the irradiance prior to insonation and \langle\rangle operation is referred to spatial averaging (over all pixels- $x$ and $y$ ).

\section{Results}

As discussed, phase distribution analysis is important to understand the behavior of the changes in the MIC signal. Figures 3(a)3(c) represent the phase distribution properties and histogram for different displacements induced by the ARF, obtained from simulations. Figure 3(a) shows that as the maximum displacement increases, average of the $\Delta$ OPL distribution does not change, but the standard deviation increases. Since the displacement is unidirectional, the OPL for some of the paths increases and for some of the paths decreases; therefore, the average $\Delta \mathrm{OPL}$ is expected to be unchanged. Figure 3(b) shows the histogram of the distribution. By applying displacement to particles in the model, the distribution of the $\triangle \mathrm{OPL}$ becomes wider. As the distribution becomes wider, the phase distribution [Figure 3(c)] becomes closer to a uniform distribution in the $0-2 \pi$ span. Therefore, the MIC signal becomes random. This is referred to as modulation saturation in the literature. ${ }^{44}$
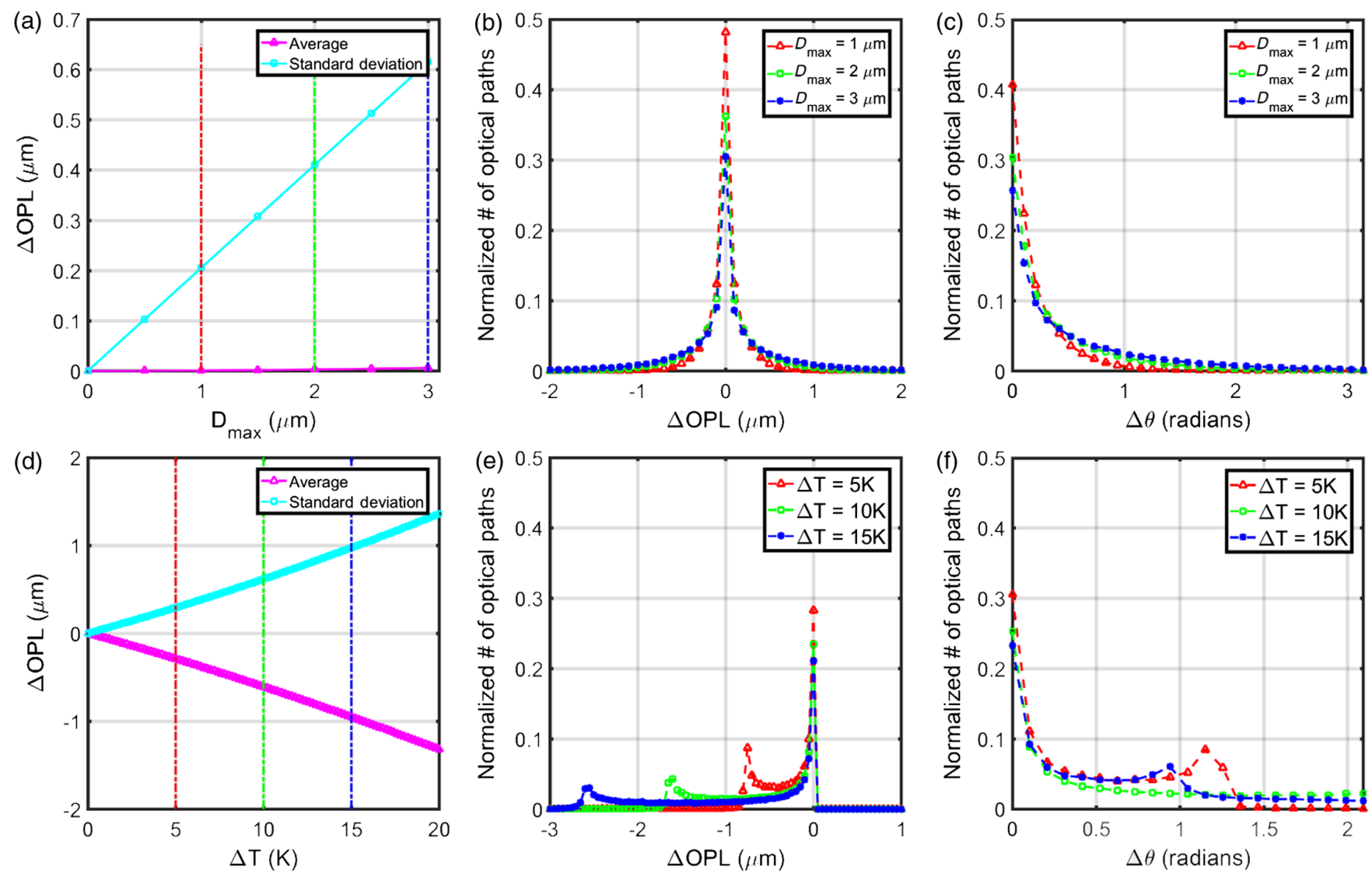

Fig. 3 (a) Average and standard deviation of changes in OPL corresponding to the displacement induced by the ARF. (b) Distribution of the OPL changes and (c) the phase distribution, for three values of $D_{\max }$. (d) Average and standard deviation of changes in OPL corresponding to changes in temperature. (e) Distribution of the OPL changes and (f) the phase distribution, for three values of $\Delta T$. 
Figures 3(d)-3(f) shows properties of the phase distribution and histogram corresponding to variation of temperature, obtained from simulations. Figure 3(d) represents average and standard deviation of the distribution of the $\Delta$ OPL. By increasing the temperature, the local index of refraction of the medium decreases resulting in negative changes in all the affected scattering paths and hence the average of the phase distribution decreases. Figure 3(e) shows the histogram of the phase distribution. As the temperature increases, the histogram shifts toward negative $\Delta$ OPL. The main peak corresponds to the number of paths that are not affected by the temperature rise (at zero). The second peak corresponds to the number of the paths that are affected by the temperature rise the most i.e., paths that go through the US focal spot. Figure 3(f) shows the phase distribution corresponding to the temperature rise.

Utilizing the phase distributions for displacement and temperature rise and Eq. (10), we can find the MIC signal variation with the presence of the US wave. Figure 4 shows the simulation results of the variation of the MIC signal as a function of temperature for two cases of no-displacement and $2 \mu \mathrm{m}$ maximum displacements. The whole process starts from state \#1 (the original state-labeled as \#1 in Fig. 4) and after the cooling process, it ends at state \#1 as well.

The experimentally recorded MIC signal for four different US exposure times and peak pressure of $2.78 \mathrm{MPa}$ is presented in Fig. 5. Note that before insonation, the MIC signal is not zero. This is due to the slight variation in the speckle pattern as a result of image acquisition settings and changes in the wavelength of the laser.

In the four different US exposure times in our experimental results, it can be seen that the change in the MIC signal from state \#1 to state \#2 corresponding to the ARF excitation process is the same, i.e., the rising edges of all four experiments are the same. The reason is that the change in the MIC signal is cause by the displacement, and displacement is induced only by the ARF. Since the US wave intensity and material stiffness remained the same for different US exposure times, the change in the MIC signal remained the same as well, reflecting the local displacement in the medium at the US focal spot.

\section{Discussion}

The MIC signal is governed by the location of the particles and property of the light source. In our simulation, we assume that during each process, particles' locations are fixed and the light source is stable. Therefore, the MIC signal does not change.

Experimental results presented in Fig. 5 illustrate that the rapid change in the MIC signal corresponding to the ARF excitation state is constant for a given peak pressure, showing that it is independent of the pulse width and only a function of the peak pressure and the medium properties. This quantity is proportional to the local displacement of the medium at the US focal spot and monitoring this value can provide the local displacement image of the medium, which potentially can be transformed into stiffness image of the medium. In our simulations, during this process we neglected the temperature rise and the displacement is modeled as the maximum displacement reached by the ARF. Hence particles are at their new fixed location. This is shown in Figs. 4(a) and 4(b) with an arrow going from state \#1 to state \#2.

During the heating process, the temperature increases and simultaneously, due to thermal diffusion, the heat spreads in the medium. This process continues till the temperature reaches
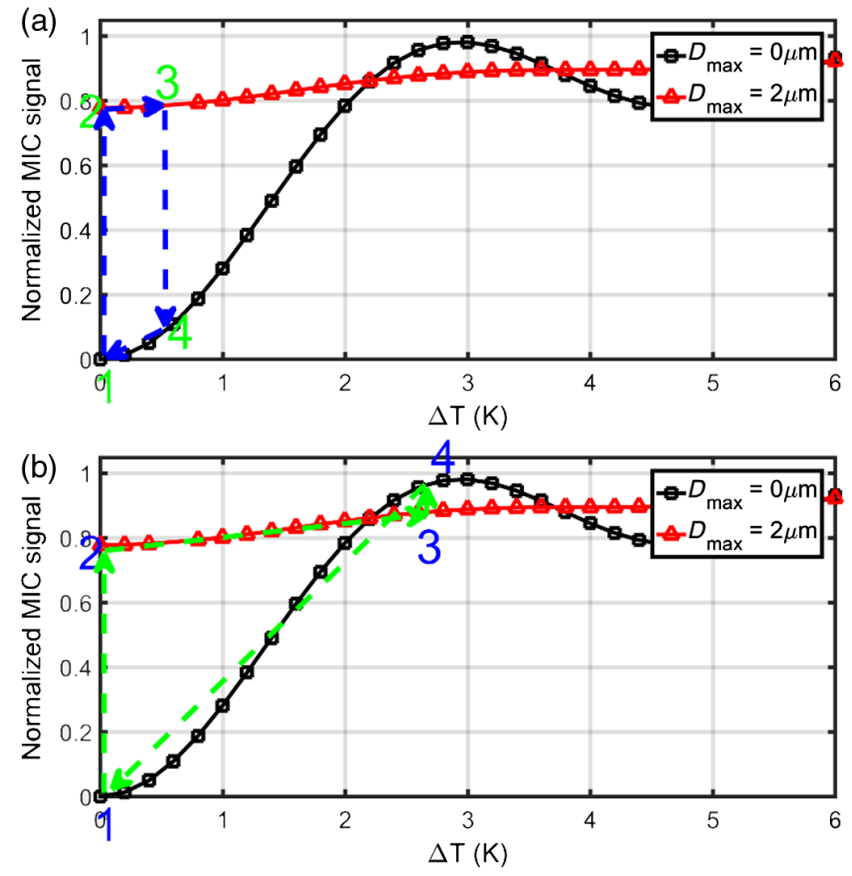

Fig. 4 Simulation results for two cases of no-displacement and $2 \mu \mathrm{m}$ maximum displacement. (a) Short US exposure time experiment (50 or $100 \mathrm{~ms}$ ) and (b) long US exposure time experiment (1 or $2 \mathrm{~s}$ ). The process begins at state \#1 as the original state followed by ARF excitation (with maximum displacement) that corresponds to the jump to state \#2 (from black curve to the red curve). From state \#2, absorbed energy gradually generates heat and changes the MIC signal to the state \#3 (on the red curve), which is followed by ARF relaxation that corresponds to the jump to the state \#4 (from red curve to the black curve). After state \#4, the MIC signal gradually goes back to the original state as the temperature gradually decreases to the original temperature (on the black curve). In the case of using a 1 or $2 \mathrm{~s}$ pulse width, the ARF relaxation corresponds to an instantaneous increase in the MIC signal.

its equilibrium point. At this moment the temperature does not change and therefore, the index of refraction and finally the MIC signal does not change. This can be seen in the longer exposure times ( 1 and $2 \mathrm{~s}$ exposure time) in the experimental results presented in Fig. 5 on the cyan curve. During this process, in our simulations particles are assumed to be at their new fixed location (reached during the ARF excitation process) and the temperature rises as in Equation 12. This is shown in Figs. 4(a) and 4(b) with an arrow going from state \#2 to state \#3. It is important to mention that with the US pulse width and the intensity we used in our experiments, temperature change does not exceed safety limits.

After the US pulse is turned off, particles move back to their original location. This implies the same amount of displacement as in ARF excitation process, but in the other direction. In simulations, this process is modeled with particles located at their original location and temperature change is neglected, hence temperature is set to be the maximum temperature reached during the heating process. This is shown in Figs. 4(a) and 4(b) with an arrow going from state \#3 to state \#4. In short US exposure times (50 and $100 \mathrm{~ms}$ ), it corresponds to a drop in the MIC signal toward the original stat [Fig. 4(a)], whereas for longer exposure times ( 1 and $2 \mathrm{~s}$ ), the MIC signal increase further more from the original state [Fig. 4(b)].

The cooling process follows after the ARF relaxation, where temperature gradually changes due to thermal conductivity of 


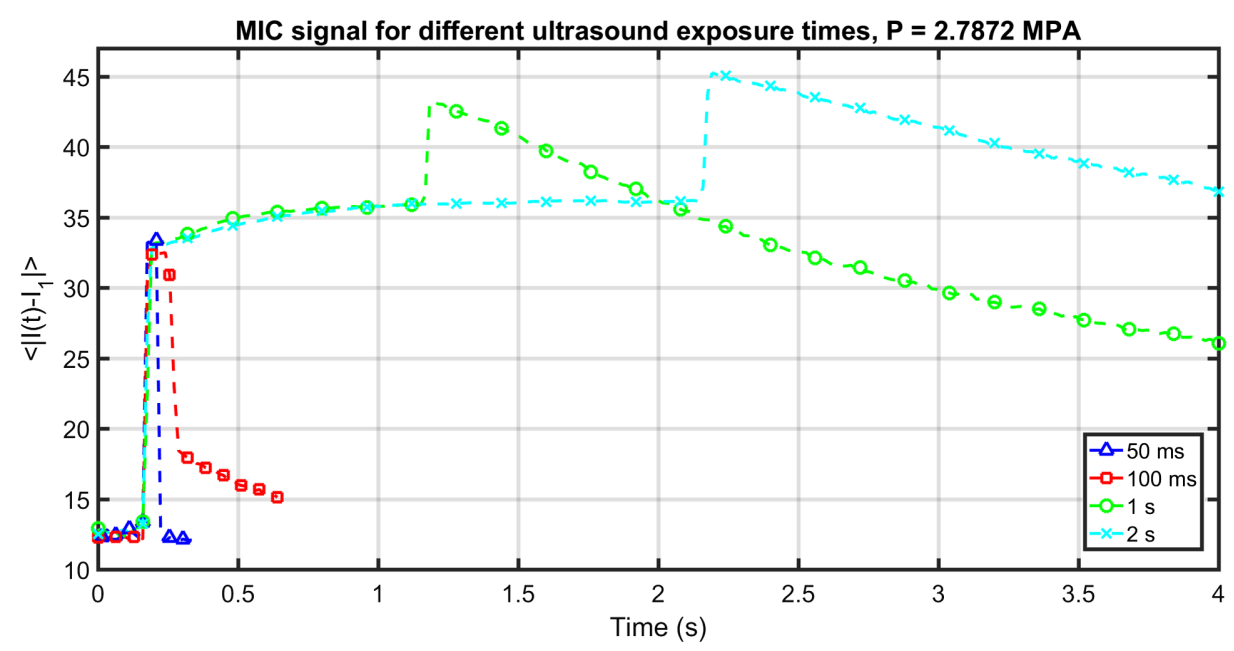

Fig. 5 Experimental results for the variation of the MIC signal for four different US exposure times with peak pressure of $2.78 \mathrm{MPa}$. The instantaneous change at the beginning of the insonation is constant for all four different US exposure times. The change in the MIC signal corresponding to the thermal effect is noticeable in longer US exposure times ( 1 and $2 \mathrm{~s}$ ). Note that the instantaneous change corresponding to the ARF relaxation process is different for each experiment. For shorter US exposure times (50 and $100 \mathrm{~ms}$ ), ARF relaxation results in change in the MIC signal toward the original state. However, for longer US exposure times, ( 1 and 2 s), ARF relaxation results in an instantaneous change further from the original state.
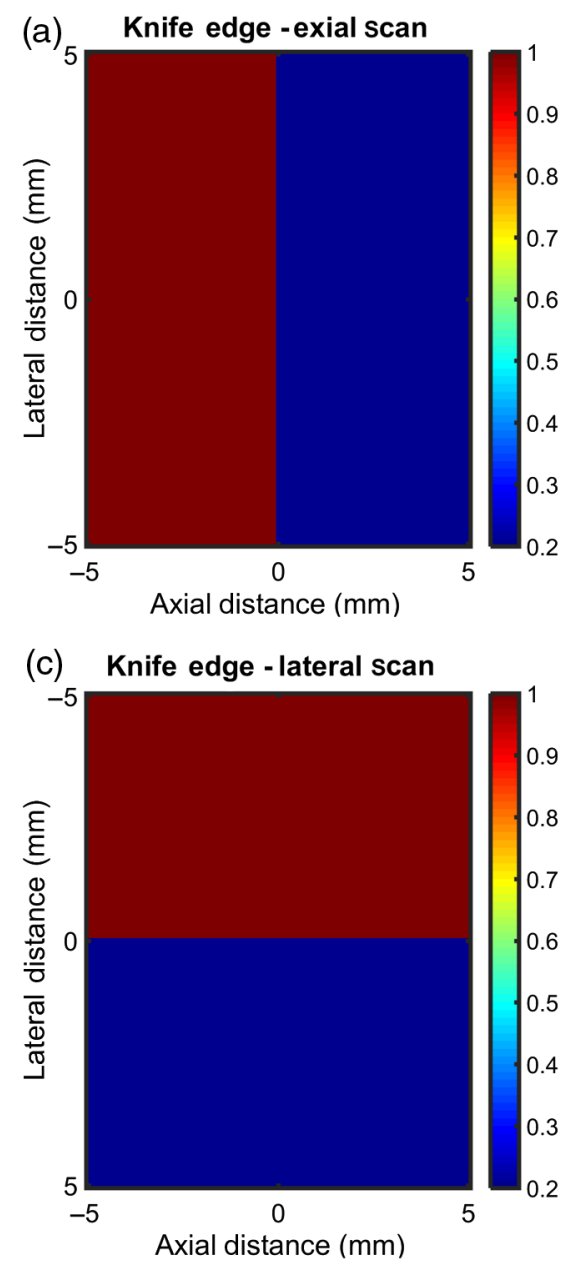

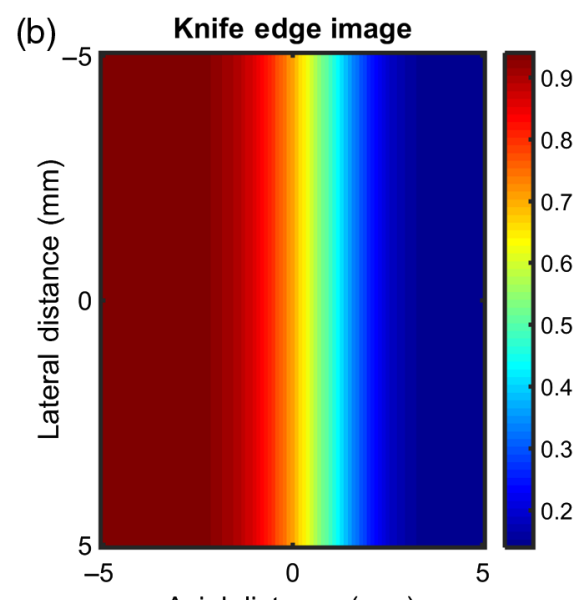

Axial distance $(\mathrm{mm})$

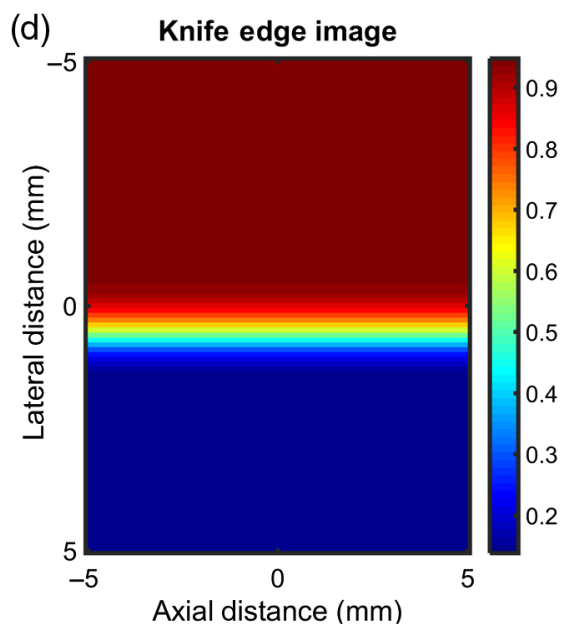

Fig. 6 Knife edge images utilizing the variation in the MIC signal. (a) and (c) Displacement map of the medium, i.e., different maximum displacements are induced in each region as the result of different stiffness in the medium. (b) and (d) Simulation results of the reconstructed displacement image utilizing the MIC signal by scanning the US focal spot in the medium. 
the temperature at the original state. This is seen in the MIC signal as a gradual change toward the MIC signal at the original state. In our simulations, the temperature change in this process is modeled as in Eq. (13). This is shown in Figs. 4(a) and 4(b) with an arrow going from state \#4 to state \#1.

The change in the MIC signal is not only a function of the displacement and/or temperature change, but also it depends on the state of the phase distribution prior to the displacement and/ or change in the temperature. In the case of the ARF excitation state, both induced displacement and the phase distribution of the prior state (original state) are the same. Hence the instantaneous change in the MIC signal is constant for all different US exposure times. However, when the ARF relaxation occurs, although it induces the same displacement, due to the temperature change that is different for different US exposure times, the phase distribution of the prior state (right before the ARF relaxation) is different for different US exposure times. Therefore, the response in the MIC signal to the ARF relaxation process is different for all different US exposure times. Experimental results show that unlike short exposure times (50 and $100 \mathrm{~ms}$ ), for long exposure times the ARF relaxation process results in further change from the original state. This has been discussed and explained with the fixed-particle Monte Carlo model. ${ }^{39,45}$
The instantaneous change in the MIC signal corresponding to the ARF excitation is constant for all US exposure times and it reflects the amount of the local displacement induced by the ARF. Figure 6 represents the simulation results to construct a displacement image based on the changes in the MIC signal corresponding to the ARF excitation process. (a) and (c) represent the induced displacement map of the model that is the result of different mechanical properties in the two regions; and (b) and (d) represent the reconstructed image of the local displacement utilizing the instantaneous change in the MIC signal.

The transition from the smaller displacement region to the larger displacement region of the medium measures the performance of the imaging system. Figure 7 represents the transition in both axial (a) and lateral (b) directions. In order to find the resolution of the image, we have utilized the $90 \%$ to $10 \%$ criteria $^{46}$ which measures the transition between the $90 \%$ to the $10 \%$ of the maximum brightness in the image. Results show that the resolution of the image is $3.3 \mathrm{~mm}$ in axial direction and $1 \mathrm{~mm}$ in the lateral direction.

To enhance resolution, higher US frequency can be utilized. Increasing the US frequency affects the signal strength. The acoustic absorption coefficient is a function of frequency. As the frequency increases, the acoustic absorption coefficient
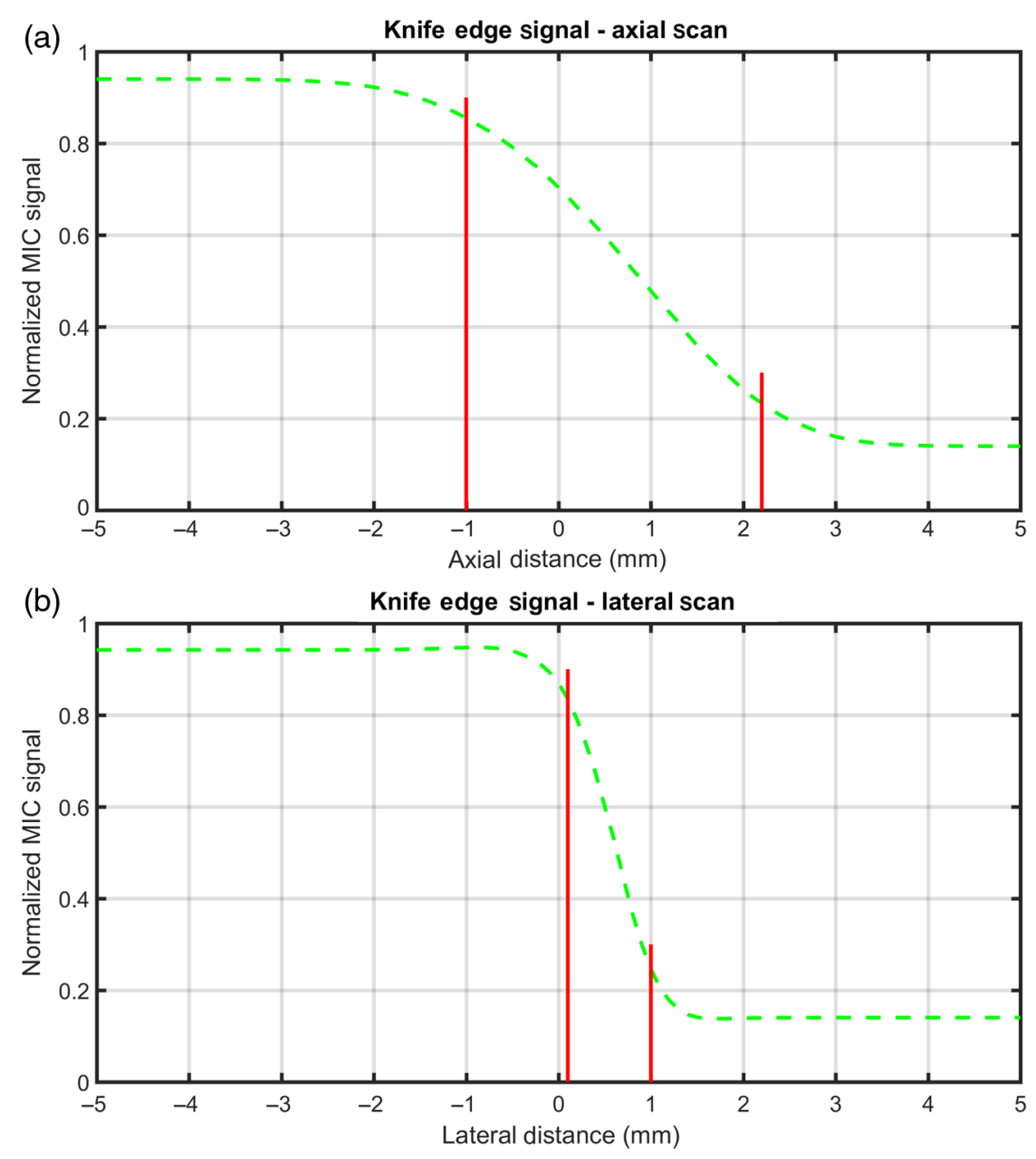

Fig. 7 Knife edge signal. The transition from the larger displacement region (higher level of the MIC signal) to the smaller displacement region of the medium (lower level of the MIC signal) represents the imaging system performance. Utilizing the $90 \%$ to $10 \%$ criteria, we can define the resolution of the system to be about $3.3 \mathrm{~mm}$ in the axial direction (a) and $1 \mathrm{~mm}$ in the lateral direction (b). 
increases as in Eq. (1). Therefore, higher US frequency results in a stronger ARF, which consequently increases the MIC signal by inducing a larger change to the OPL of the tagged scattering paths. However, higher US frequency results in a smaller US focal spot. Therefore, smaller number of scattering paths can be tagged utilizing a higher US frequency, which results in a weaker signal. The frequency dependence of the signal strength has not been studied in this work.

It is important to add that when a $1 \mathrm{MHz}$ US is present at pressures on the order of $1 \mathrm{MPa}$ as in this work, inertial cavitation is a strong possibility. ${ }^{25}$ The presence of inertial cavitation in the gel would have two possible effects with regard to the radiation force and the movement of the gel. First, the presence of bubbles would enhance the radiation force, thus enhancing the observed instantaneous change in the MIC signal. Second, the presence of inertial cavitation would result in enhanced heating of the gel phantom. ${ }^{25}$ As with the radiation force, the enhanced bubble heating would have the effect of enhancing the observed change in the MIC signal during the heating process. Taken together, the net effect of cavitation, if it is present, would be to enhance the MIC signal observation.

\section{Conclusion}

In this paper, we have introduced an approach to tag light with US similar to UOT. The OPL change induced to the tagged light with ARF displacement is in the order of microns, whereas the OPL induced in the UOT regime is in the order of nanometers. Therefore, the signal obtained utilizing the ARF to tag the light is about three orders of magnitude stronger than the modulated signal in UOT. This method makes the easier detection possible with a CCD camera.

Experimental results show that displacement and thermal effects in the ARF regime happen in different time scales. The displacement occurs in timescale of millisecond and thermal effect occurs in timescale of seconds. Monitoring the MIC signal can separately show two effects.

We demonstrated this technique through 2-D fixed-particle Monte Carlo simulations. In our Monte Carlo model, paths are defined by "photon" trajectories and the results are represented by a complex amplitude function. The magnitude of this function is determined by scattering and absorption, and the phase is determined by OPL. The phase distribution defined as the changes to the OPL of the scattering paths are used to calculate the MIC signal in simulations. Simulation results are in well agreement with experimental results. Suggesting that fixed-particle Monte Carlo is able to provide the coherence required to model the speckle pattern analysis.

We have utilized the rapid change in the MIC signal (obtained from simulation) at the beginning of the insonation to reconstruct a displacement map of the medium as shown in Fig. 6. The displacement image can potentially be converted to a stiffness image of the medium. The results show that the image resolution in axial direction is about 3.3 and $1 \mathrm{~mm}$ in lateral direction.

\section{Disclosures}

The authors have nothing to disclose.

\section{References}

1. J. Selb, L. Pottier, and A. C. Boccara, "Nonlinear effects in acoustooptic imaging," Opt. Lett. 27(11), 918-920 (2002).
2. G. Yao and L. V. Wang, "Theoretical and experimental studies of ultrasound-modulated optical tomography in biological tissue," Appl. Opt. 39(4), 659-664 (2000).

3. M. Kempe et al., "Acousto-optic tomography with multiply scattered light," J. Opt. Soc. Am. A 14(5), 1151-1158 (1997).

4. K. Maslov et al., "Optical-resolution photoacoustic microscopy for in vivo imaging of single capillaries," Opt. Lett. 33(9), 929-931 (2008).

5. J. J. Niederhauser et al., "Combined ultrasound and optoacoustic system for real-time high-contrast vascular imaging in vivo," IEEE Trans. Med. Imaging 24(4), 436-440 (2005).

6. S. L. Jacques and S. J. Kirkpatrick, "Acoustically modulated speckle imaging of biological tissues," Opt. Lett. 23(11), 879-881 (1998).

7. J. McKinney et al., "Characterization and imaging in optically scattering media by use of laser speckle and a variable-coherence source," Opt. Lett. 25(1), 4-6 (2000).

8. E. Granot et al., "Detection of inhomogeneities with ultrasound tagging of light," J. Opt. Soc. Am. A 18(8), 1962-1967 (2001).

9. P. Lai, R. A. Roy, and T. W. Murray, "Quantitative characterization of turbid media using pressure contrast acousto-optic imaging," Opt. Lett. 34(18), 2850-2852 (2009).

10. L. V. Wang, "Mechanisms of ultrasonic modulation of multiply scattered coherent light: an analytic model," Phys. Rev. Lett. 87(4), 043903 (2001).

11. G. Yao, S. Jiao, and L. V. Wang, "Frequency-swept ultrasoundmodulated optical tomography in biological tissue by use of parallel detection," Opt. Lett. 25(10), 734-736 (2000).

12. L. V. Wang and G. Ku, "Frequency-swept ultrasound-modulated optical tomography of scattering media," Opt. Lett. 23(12), 975-977 (1998).

13. L. Wang and X. Zhao, "Ultrasound-modulated optical tomography of absorbing objects buried in dense tissue-simulating turbid media," Appl. Opt. 36(28), 7277-7282 (1997).

14. L. Wang, S. L. Jacques, and X. Zhao, "Continuous-wave ultrasonic modulation of scattered laser light to image objects in turbid media," Opt. Lett. 20(6), 629-631 (1995).

15. W. Leutz and G. Maret, "Ultrasonic modulation of multiply scattered light," Phys. B 204(1), 14-19 (1995).

16. J. L. Hollmann et al., "Analysis and modeling of an ultrasound-modulated guide star to increase the depth of focusing in a turbid medium," J. Biomed. Opt. 18(2), 025004 (2013).

17. J. L. Hollmann and C. A. DiMarzio, "Modeling optical phase conjugation of ultrasonically encoded signal utilizing finite-difference timedomain simulations," Proc. SPIE 8589, 85890E (2013).

18. J. L. Hollmann et al., "Diffusion model for ultrasound-modulated light," J. Biomed. Opt. 19(3), 035005 (2014).

19. S. Leveque et al., "Ultrasonic tagging of photon paths in scattering media: parallel speckle modulation processing," Opt. Lett. 24(3), 181-183 (1999).

20. F. A. Duck, "Nonlinear acoustics in diagnostic ultrasound," Ultrasound Med. Biol. 28(1), 1-18 (2002).

21. K. Nightingale et al., "Acoustic radiation force impulse imaging: in vivo demonstration of clinical feasibility," Ultrasound Med. Biol. 28(2), 227235 (2002).

22. M. Friedrich-Rust et al., "Liver fibrosis in viral hepatitis: noninvasive assessment with acoustic radiation force impulse imaging versus transient elastography," Radiology 252(2), 595-604 (2009).

23. K. Nightingale, "Acoustic radiation force impulse (ARFI) imaging: a review," Curr. Med. Imaging Rev. 7(4), 328-339 (2011).

24. K. Nightingale, S. McAleavey, and G. Trahey, "Shear-wave generation using acoustic radiation force: in vivo and ex vivo results," Ultrasound Med. Biol. 29(12), 1715-1723 (2003).

25. R. G. Holt and R. A. Roy, "Measurements of bubble-enhanced heating from focused, mhz-frequency ultrasound in a tissue-mimicking material," Ultrasound Med. Biol. 27(10), 1399-1412 (2001).

26. R. Clarke and G. Ter Haar, "Temperature rise recorded during lesion formation by high-intensity focused ultrasound," Ultrasound Med. Biol. 23(2), 299-306 (1997).

27. K. Hynynen, "The threshold for thermally significant cavitation in dog's thigh muscle in vivo," Ultrasound Med. Biol. 17(2), 157-169 (1991).

28. D. Leger, E. Mathieu, and J. Perrin, "Optical surface roughness determination using speckle correlation technique," Appl. Opt. 14(4), 872-877 (1975). 
29. I. Yamaguchi, "A laser-speckle strain gauge," J. Phys. E Sci. Instrum. 14(11), 1270-1273 (1981).

30. G. Xiong et al., "Particle-fixed Monte Carlo model for optical coherence tomography," Opt. Express 13(6), 2182-2195 (2005).

31. G. Torr, "The acoustic radiation force," Am. J. Phys 52(5), 402-408 (1984).

32. W. 1. M. Nyborg, "Acoustic streaming," Phys. Acoust. Princ. Methods 11, 265 (1965).

33. H. Starritt, F. Duck, and V. Humphrey, "Forces acting in the direction of propagation in pulsed ultrasound fields," Phys. Med. Biol. 36(11), 1465-1474 (1991).

34. K. R. Nightingale et al., "On the feasibility of remote palpation using acoustic radiation force," J. Acoust. Soc. Am. 110(1), 625-634 (2001).

35. K. J. Parker, "The thermal pulse decay technique for measuring ultrasonic absorption coefficients," J. Acoust. Soc. Am. 74(5), 1356-1361 (1983).

36. A. N. Bashkatov and E. A. Genina, "Water refractive index in dependence on temperature and wavelength: a simple approximation," Proc. SPIE 5068, 393-395 (2003).

37. J. C. Dainty, Laser Speckle and Related Phenomena, Topics in Applied Physics, Vol. 9, p. 298, Springer Berlin Heidelberg, Berlin, Heidelberg (1975).

38. J. W. Goodman, "Statistical properties of laser speckle patterns," in Laser Speckle and Related Phenomena, pp. 9-75, Springer (1975).
39. A. Vakili et al., "Acoustic effects analysis utilizing speckle pattern with fixed-particle Monte Carlo," Proc. SPIE 9713, 971309 (2016).

40. C. F. Bohren and D. R. Huffman, "Absorption and scattering by a sphere," in Absorption and Scattering of Light by Small Particles, John Wiley \& Sons (2008).

41. J. W. Goodman, Speckle Phenomena in Optics: Theory and Applications, Roberts and Company Publishers, Englewood, Colorado (2007).

42. S. J. Kirkpatrick, D. D. Duncan, and E. M. Wells-Gray, "Detrimental effects of speckle-pixel size matching in laser speckle contrast imaging," Opt. Lett. 33(24), 2886-2888 (2008).

43. G. Mitic et al., "Time-gated transillumination of biological tissues and tissue like phantoms," Appl. Opt. 33(28), 6699-6710 (1994).

44. L. V. Wang, "Mechanisms of ultrasonic modulation of multiply scattered coherent light: a Monte Carlo model," Opt. Lett. 26(15), 11911193 (2001).

45. A. Vakili et al., "Imaging beyond scattering limits utilizing ARF as a guidestar," Proc. SPIE 10070, 100700W (2017).

46. P. J. Dwyer, C. A. DiMarzio, and M. Rajadhyaksha, "Confocal theta line-scanning microscope for imaging human tissues," Appl. Opt. 46(10), 1843-1851 (2007).

Biographies for the authors are not available. 